\title{
The Clinical Significance of High Antimicrobial Resistance in Community-Acquired Urinary Tract Infections
}

\author{
Maria G. Zavala-Cerna $\mathbb{D}^{1},{ }^{1}$ Midrori Segura-Cobos, ${ }^{1}$ Ricardo Gonzalez, ${ }^{2}$ \\ Isidro G. Zavala-Trujillo, ${ }^{3}$ Silvia F. Navarro-Perez $\mathbb{D}^{1},{ }^{1}$ Jose A. Rueda-Cruz, ${ }^{4}$ \\ and Fernando A. Satoscoy-Tovar ${ }^{4}$ \\ ${ }^{1}$ Immunology Department, International Program of Medicine, Universidad Autonoma de Guadalajra, Guadalajara, Jal, Mexico \\ ${ }^{2}$ Facultad de Biología, Instituto de Ciencias Exactas y Terrestres, Universidad Autonoma de Guadalajara, Guadalajara, \\ Jal, Mexico \\ ${ }^{3}$ Infectious Disease Division, Hospital Angel Leaño, Universidad Autonoma de Guadalajara, Guadalajara, Jal, Mexico \\ ${ }^{4}$ Microbiology Department, Unidad de Patología Clínica, Guadalajara, Jal, Mexico
}

Correspondence should be addressed to Maria G. Zavala-Cerna; g_zavala_78@hotmail.com

Received 23 May 2019; Revised 21 April 2020; Accepted 13 May 2020; Published 5 June 2020

Academic Editor: Matthias Karrasch

Copyright ( 2020 Maria G. Zavala-Cerna et al. This is an open access article distributed under the Creative Commons Attribution License, which permits unrestricted use, distribution, and reproduction in any medium, provided the original work is properly cited.

\begin{abstract}
Background. Urinary tract infections (UTIs) affect up to 150 million individuals annually worldwide, mainly due to Escherichia coli (E. coli) and Klebsiella. The emergence and spread of multidrug-resistant (MDR) bacteria are increasing, representing one of the biggest threats for human health. The objective of our study was to describe antimicrobial patterns of resistance and identify risk factors associated with MDR uropathogens. Methods. We conducted a cross-sectional study in 296 patients with communityacquired UTI who underwent clinical and microbiologic analysis, and clinical associations to MDR uropathogens were investigated. Findings. Microbiological analysis included E. coli (55\%), ESBL-E. coli (26\%), Enterococcus (6\%), Klebsiella (5\%), and others (8\%). Higher frequencies of MDR bacteria were found among ESBL-E. coli, with resistance to ampicillin (100\%), ceftriaxone (96\%), gentamicin (57\%), ciprofloxacin (89\%), and TMP/SMX (53\%). However, they were sensitive to fosfomycin (6.6\%), nitrofurantoin (1.3\%), and carbapenems (0\%). Fosfomycin MIC90 for ESBL-E. coli was $5.78 \mu \mathrm{g} / \mathrm{mL}$. The only clinical variable with significant association to ESBL producers was the presence of comorbidities: hypertension and type 2 diabetes mellitus with an OR $(95 \% \mathrm{CI})$ of $2.5(1.3-4.9)(p<0.01)$ and $2.8(1.2-6.7)(p<0.05)$, respectively. Conclusions. In the majority of cases, resistance rates to commonly prescribed antimicrobials in UTIs were high, except for fosfomycin, nitrofurantoin, and carbapenems. To provide appropriate treatment, both the identification of risk factors and the uropathogen would be important. An active surveillance in UTIs in the community is required since the proportion of ESBL producers is increasing.
\end{abstract}

\section{Introduction}

Urinary tract infections (UTIs) are defined as the presence of classical signs and/or symptoms and urine culture demonstrating the presence of known uropathogens above the given threshold ( $>100 \mathrm{cfu} / \mathrm{ml}$ urine to $100,000 \mathrm{cfu} / \mathrm{ml}$ urine) [1]. Although this combination is not always present, it is estimated that close to $20 \%$ of women can manifest symptoms without positive urine cultures and significant numbers of bacteria can be found in asymptomatic individuals [2,3]. UTIs are classified as either complicated or uncomplicated; the first depends on the presence of anatomical or functional urological abnormalities, pregnancy, immunosuppression, and signs and symptoms related to tissue invasion or systemic affection [4]. UTIs affect up to 150 million individuals annually worldwide [5]; incidence varies across continents with reports of 2,400 per 100,000 women annually in Europe and over 8 million annual cases in the US. This amount of cases provided data to estimate that 1 out of 3 women will develop a UTI requiring antibiotic 
treatment by age of 24, and 1 out of 2 women will develop at least one episode of UTIs during their lifetime [6]. Successful bacterial invasion of the urinary tract depends on several factors, either attributable to the host, such as barrier defense mechanisms, hormone regulation of the immune system, and changes in the genital microbiota, or factors attributable to the pathogen itself such as inoculum size, virulence factors that are aimed at the adhesion or invasion of the urinary tract epithelia, and the formation of biofilms that confer protection against host immune response or antibiotic treatment [7]. Common uropathogens that cause community-associated UTIs include Escherichia coli (E. coli), Klebsiella pneumoniae, and Enterococci, with the first being the primary causative agent (80\%) in most studies [8]. Furthermore, among E. coli isolates, it has been found that from $1.6 \%$ to $3.9 \%$ are producers of extended spectrum-beta lactamases (ESBLs) in outpatient settings reported in France and the United States, respectively $[9,10]$, and in our own previous experience, but with a different approach, ESBLs can be found in up to $69.5 \%$ in the outpatient setting [11]. The 2010 IDSA clinical guidelines for treatment of uncomplicated UTIs recommend selecting one of the following regimens of treatment: (1) nitrofurantoin monohydrate/ macrocrystals $100 \mathrm{mg}$ twice daily for 5 days; (2) TMP/SMX 160/180 mg twice daily for 3 days; (3) fosfomycin $3 \mathrm{mg}$ as a single dose; (4) pivmecillinam $400 \mathrm{mg}$ twice daily [12]. Uropathogens in the community setting have developed high rates of resistance, specially to quinolones carbapenems and third-generation cephalosporins, due to bacterial production of extended-spectrum-beta lactamases (ESBLs) [13], carbapenemases [14], and biofilm production, which allows the bacteria to withstand hostile environmental conditions and makes them capable of causing a broad range of chronic diseases [15]. The mechanisms for acquisition of these proteins can be either by de novo mutations or mobile genetic elements carrying resistance genes [16]. The emergence and spread of multidrug-resistant (MDR) bacteria is increasing worldwide, defined as resistance to at least one agent of three or more antibiotic classes, and represents one of the biggest health threats for human health, not just in the recent years, but will be a major issue in the coming decades, all the way to a devastating problem in 2050, unless we find alternatives for treatment [17]. Attributable factors for this phenomenon include limited therapeutic options, limited diagnostic facilities, inappropriate prescription practices, inadequate patient education, unauthorized sales of antimicrobials, and lack of appropriate functionating drug regulatory mechanisms [18]. While several comorbidities increase susceptibility to UTIs, most of them occur in otherwise healthy women; therefore, the objective of this work was to describe antimicrobial patterns of resistance in our community, as well as the identification of risk factors associated with the presence of multiresistant pathogens causing UTIs.

\section{Methods}

We conducted a cross-sectional study, in the occupational medicine clinic at "Unidad de Patologia Clinica" in
Guadalajara, Jal., Mexico. The study was reviewed and approved by the Universidad Autonoma de Guadalajara Health Sciences Center Institutional Review Board prior to initiation.

2.1. Inclusion of Subjects. For the present study, we recruited subjects from the occupational clinic (provides service for several companies that have facilities in the state of Jalisco), as well as patients referred from private clinics and public health systems in Guadalajara, Jalisco.

Eligible subjects were assessed for inclusion criteria and were invited to participate in the study after explanation of the informed consent and acceptance to participate in the study.

Subjects were assessed by general practice physicians from Universidad Autonoma de Guadalajara, in the occupational clinic facilities. Inclusion criteria were subjects with suspicion of a UTIs by their treating physician, subjects $>18$ years old, capable of answering questionnaires, and voluntarily accepted participation in the study. Exclusion criteria were subjects that declared the intake of broadspectrum antibiotics or being in a hospital or nursery home 3 days prior to the inclusion.

Predesigned questionnaires were used to obtain clinical data relevant for urinary tract infections. All included patients underwent urine sample collection. Cases of urinary tract infections were defined as the presence of at least one sign or symptom previously associated with the disease in combination with the presence of $10^{3}$ bacteria $/ \mathrm{mL}$ or as asymptomatic bacteriuria with the sole presence of $10^{5}$ bacteria/mL in the urine sample without clinical manifestations. In all cases, urine samples were collected by clean catch method; samples were conserved at room temperature before they were analyzed (no more than 30 minutes). All samples underwent urinalysis and culture.

2.2. Microbiological Analyses. We used standard techniques for culture and pathogen identification [19]. Bacteria susceptibility testing to antimicrobial drugs were performed by microdilution methodology (VITEK 2 XL @ Biomerieux), with panels comprising different concentrations for antibiotic drugs, except for fosfomycin. Inhibition for each isolate was defined as susceptible, intermediate, or resistant, according to the guidelines of Clinical and Laboratory Standards Institute (CLSI) [20]. To identify ESBL producer strains, by phenotypic conformation, we used the AST-N286 card containing cefepime $(1 \mathrm{mg} / \mathrm{mL})$, cefotaxime $(0.5 \mathrm{mg} /$ $\mathrm{mL})$, ceftazidime $(0.5 \mathrm{mg} / \mathrm{mL})$, cefepime/clavulanic acid (1/ $10 \mathrm{mg} / \mathrm{mL})$, cefotaxime/clavulanic acid $(0.5 / 4 \mathrm{mg} / \mathrm{mL})$, and ceftazidime/clavulanic acid $(0.5 / 4 \mathrm{mg} / \mathrm{mL})$ as recommended by the CLSI. Bacterial growth at or above the screening antibiotic concentration for all penicillins and cephalosporins was confirmed as ESBL production.

The in vitro susceptibility to fosfomycin was measured by broth dilution and commercially available disk diffusion containing $200 \mu \mathrm{g}$ of fosfomycin and $50 \mu \mathrm{g}$ of G6P. The interpretation of fosfomycin susceptibility in $E$. coli was performed using the CLSI 2012 guidelines: susceptible 
$(\leq 64 \mathrm{mg} / \mathrm{mL})$, intermediate $(128 \mathrm{mg} / \mathrm{mL})$, or resistant ( $\geq 256 \mathrm{mg} / \mathrm{mL}$ ) [20]. MIC and minimum bactericidal concentration $(\mathrm{MBC})$ of fosfomycin to microbial strains was determined by broth dilution as well [21]. In brief, Mueller-Hinton agar (MHA) medium was adjusted to different concentrations of fosfomycin $(5-160 \mu \mathrm{g} / \mathrm{mL})$ and glucose-6phosphate (G6P) was added; then, bacterium inoculum was introduced, previously quantified $(\mathrm{O} . \mathrm{D} 600 \mathrm{~nm}=0.1$; approximately $1 \times 108$ per $\mathrm{mL}^{-1}$ ), and diluted $1: 10$ in saline solution. Petri dishes were incubated $18-24 \mathrm{hrs}$ at $37^{\circ} \mathrm{C}$. After this time, bacterial colonies were evaluated according to their growth and compared with respect to the control. Control plaques were used for bacterial growth in the absence of antibiotics to determine both bacterial growth and viability.

2.3. Statistical Analysis. For descriptive purposes, categorical variables are presented as numbers and percentages and continuous variables with mean \pm standard deviation, unless otherwise indicated. For inference purposes, categorical variables were compared using $\chi^{2}$ analysis, and continuous variables with normal distributions were compared using Student's $t$-test. For nonnormally distributed variables, we used Fisher's exact test. To test differences among multiple groups of bacteria for continuous variables, we used a 1-way analysis of variance (ANOVA) with Bonferroni multiple comparison test. Except for the age, the distribution of variables was non-Gaussian (Shapiro-Wilk's test, $p>0.1$ ). For multiple variable analysis, we used logistic regression. The hypothesis was tested at 95\% confidence interval (CI), two-sided, and the level of type I error was set at $a=0.05$. All statistical analyses were done using STATA/IC 15.1 software.

\section{Results}

After 12 months, a total of 500 subjects were included in the study; 29 patients were excluded due to sample or clinical information loss. From 471 subjects, we were able to confirm positive urine cultures in 296 cases. From these, 247 were confirmed cases of UTIs with both clinical symptoms and the presence of $10^{3} \mathrm{CFU} / \mathrm{mL}$, and in 49 cases, the urine culture was positive with up to $\geq 10^{5} \mathrm{CFU} / \mathrm{mL}$, but there was absence of typical clinical manifestations. From these 296 confirmed cases of community-acquired UTIs and asymptomatic bacteriuria, the mean age was $54 \pm 19$ years; 262 (89\%) were women; of these, 130 (50\%) were postmenopausal and $13(5 \%)$ were pregnant. None of the subjects reported awareness of an anatomical/functional abnormality in the urinary tract or an immunosuppressive condition.

The most common symptom reported was pollakiuria in 228 (77\%), and the least common was fever in 47 (16\%). We found that $83(28 \%)$ patients referred at least one previous episode of a UTI in the current year that required the use of antibiotic treatment. During interview, we asked for the presence of comorbidities; the most frequent was hypertension in $80(27 \%)$ of patients, followed by diabetes and hypothyroidism. Table 1 enlists clinical variables of cases with UTIs.
TABle 1: Clinical and demographic variables of 296 patients with community-acquired urinary tract infection and asymptomatic bacteriuria.

\begin{tabular}{lc}
\hline Variable & Mean \pm SD or $n(\%)$ \\
\hline Age & $54 \pm 19$ \\
Feminine gender & $262(89)$ \\
Menopause & $130(44)$ \\
Pregnant & $13(4)$ \\
Multiparous ( $\geq 2$ births $)$ & $128(43)$ \\
Previous UTIs $(\geq 1)$ & $83(28)$ \\
\hline Signs and symptoms & $n(\%)$ \\
Pollakiuria & $228(77)$ \\
Change in urine color & $187(63)$ \\
Dysuria & $181(61)$ \\
Urinary urgency & $170(57)$ \\
Suprapubic pain & $143(48)$ \\
Hematuria & $48(16)$ \\
Fever & $47(16)$ \\
General malaise & $22(7)$ \\
\hline Comorbidities & $n(\%)$ \\
Hypertension & $66(22)$ \\
Type 2 diabetes mellitus & $25(8)$ \\
Hypothyroidism & $23(8)$ \\
\hline
\end{tabular}

After these observations, it was established that from 247 cases of UTI, 56 (23\%) were complicated, either by the presence of one of the following or a combination in both pregnancy and fever.

With respect to isolated pathogens being the causative agents of community-acquired UTIs in our study, the most frequently found was E. coli $(n=240)$, followed by Enterococcus faecalis $(n=18)$, Klebsiella pneumoniae $(n=14)$, Staphylococcus sp. $(n=7)$, Pseudomonas aeruginosa $(n=6)$, Proteus mirabilis $(n=4)$, Citrobacter freundii $(n=3)$, Candida albicans $(n=2)$, Serratia marcescens $(n=1)$, and Streptococcus agalactiae $(n=1)$. From the E. coli isolates, 76 (25.6\%) were classified as ESBL producers.

Antimicrobial resistance was tested to commonly prescribed antibiotic drugs including beta-lactams (penicillin, cephalosporins, monobactams, and carbapenems), macrolides, aminoglycosides, sulfonamides, phosphonates, nitro derivatives, and quinolones. Depending on susceptibility patterns, different numbers of antibiotics were tested for each pathogen.

Resistance rate to antimicrobials, reported in more than $20 \%$ of E. coli isolates, was reported to ampicillin, TMP/ SMX, and ciprofloxacin. Importantly, ESBL- E. coli was multidrug resistant (MDR) with high resistance to ampicillin $(100 \%)$, ceftriaxone (96\%), gentamicin (57\%), ciprofloxacin (89\%), and TMP/SMX (53\%). On the contrary, low resistance was identified to fosfomycin (6.6\%), nitrofurantoin $(1.3 \%)$, and carbapenems $(0 \%)$. The highest resistance rates to nitrofurantoin were found among Pseudomonas and Proteus isolates. Frequencies of most common isolated pathogens and their antimicrobial resistances are represented in Figure 1, taking into consideration guidelines for treatment of community-associated UTIs, except for pivmecillinam, which is not yet available in 

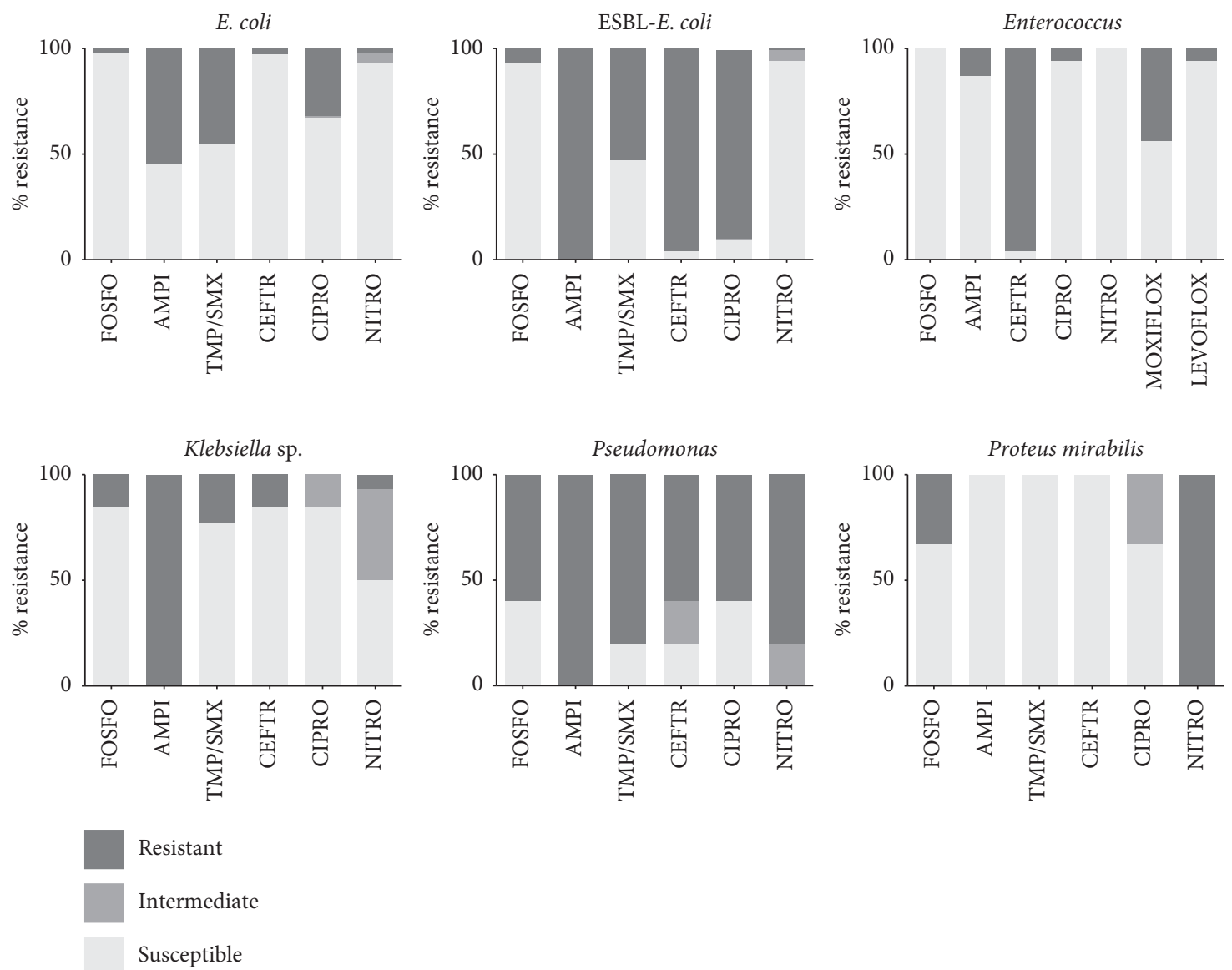

FIGURE 1: Resistance patterns in isolated uropathogens from community-associated uncomplicated cystitis to optimal treatment options according to IDSA guidelines and the European society for microbiology and infectious diseases.

TABLE 2: Randomly selected UPEC strains to determine MIC and BIC to fosfomycin.

\begin{tabular}{lccccccc}
\hline & $n$ & $R$ & $\begin{array}{c}I \\
n(\%)\end{array}$ & $S$ & MIC50 $(\mu \mathrm{g} / \mathrm{mL})$ & $\mathrm{MIC} 90(\mu \mathrm{g} / \mathrm{mL})$ & $\mathrm{MBC}(\mu \mathrm{g} / \mathrm{mL})$ \\
\hline Non-ESBL UPEC & 24 & $2(8)$ & $0(0)$ & $22(92)$ & 3.12 & 5.62 & 5.78 \\
ESBL UPEC & 24 & $4(17)$ & $0(0)$ & $20(83)$ & 3.21 & - & 63.75 \\
Klebsiella pneumoniae & 4 & $4(100)$ & $0(0)$ & $0(0)$ & - & $>160$ \\
\hline
\end{tabular}

$R$ : resistant, $I$ : intermediate, $S$ : sensible, MIC: minimal inhibitory concentration, and MBC: minimal bactericidal concentration.

Mexico. For additional and more precise information, see Supplementary Table 1.

Forty-eight strains of uropathogenic Escherichia coli (UPEC) and four strains of Klebsiella pneumoniae were randomly selected to determine the degree of susceptibility to fosfomycin. Twenty-four UPEC strains were ESBL producers, while the remaining strains were non-ESBL producers. The MIC90 of fosfomycin for the ESBL-UPEC and non-ESBL-UPEC strains was $5.78 \mu \mathrm{g} / \mathrm{ml}$ and $5.62 \mu \mathrm{g} / \mathrm{ml}$, respectively. In addition, significant differences were observed in the MBC of fosfomycin for the ESBL-UPEC strains $(63.75 \mu \mathrm{g} / \mathrm{ml})$, with respect to the non-ESBL-UPEC strains $(44.32 \mu \mathrm{g} / \mathrm{ml})$. Of the fifty-two strains analyzed, only ten were resistant to fosfomycin (four strains of Klebsiella pneumoniae and six UPEC strains) at a maximum concentration of $160 \mu \mathrm{g} / \mathrm{ml}$; complete information is provided in Table 2.
When analyzing the urinalysis results, according to the uropathogen, we found significant differences for the presence of nitrites and bacteria (Table 3 ). We found that an acidic $\mathrm{pH}$ (5.2) was significantly different for UTIs due to Klebsiella after Bonferroni multiple comparison test ( $p=0.015)$. Cases due to Proteus seem to have a more basic $\mathrm{pH}$. However, we only had a few cases, and it was not included in the statistical analysis. Almost all samples had leukocytes (94.7\%) and leukocyte esterase (88.6\%) irrespective of the uropathogen.

To identify clinical variables associated with the presence of ESBL- E. coli, we performed a multivariate analysis and found that the only clinical variable with significant association to the presence of ESBL producers was the presence of comorbidities, including hypertension and diabetes; both exhibited a significant association with an OR (IC95\%) of $2.5(1.3-4.9)(p<0.01) \quad$ for hypertension and 
TABLE 3: Urinalysis findings in patients with UTIs according to the causative agent.

\begin{tabular}{|c|c|c|c|c|c|}
\hline Urinalysis finding & E. $\operatorname{coli}(n=164)$ & ESBL-E. coli $(n=76)$ & Enterococcus $(n=18)$ & Klebsiella $(n=14)$ & $p$ value \\
\hline $\mathrm{pH}$ mean $\pm \mathrm{SD}$ & $6.04 \pm 0.7$ & $5.8 \pm 0.9$ & $5.7 \pm 0.8$ & $5.2 \pm 0.6$ & 0.415 \\
\hline Positive nitrites $n(\%)$ & $140(85)$ & $33(43)$ & $1(6)$ & $0(0)$ & 0.002 \\
\hline Positive proteins $n(\%)$ & $46(34)$ & $12(20)$ & $5(29)$ & $2(14)$ & 0.154 \\
\hline Positive hemoglobin $n(\%)$ & $26(16)$ & $9(12)$ & $2(11)$ & $3(21)$ & 0.798 \\
\hline $\mathrm{LE}(\mu \mathrm{L})$ median $(\min -\max )$ & $75(0-500)$ & $100(0-500)$ & $50(0-500)$ & $50(25-500)$ & 0.745 \\
\hline WBCs (HPF) median (min-max) & $75(0-500)$ & $38(1-800)$ & $30(2-466)$ & $27(10-315)$ & 0.993 \\
\hline RBCs (HPF) median (min-max) & $1(0-917)$ & $12(0-550)$ & $2(0-53)$ & $0.5(0-7)$ & 0.558 \\
\hline Positive bacteria $n(\%)$ & $62(58)$ & $30(28)$ & $4(22)$ & $11(79)$ & 0.048 \\
\hline
\end{tabular}

$2.8(1.2-6.7)(p<0.05)$ for diabetes. None of the other clinical variables resulted in significant association to ESBLE. coli, such as older age, feminine gender, pregnancy, menopause, previous UTIs, or hypothyroidism. After adjusting for confounding in a multiple variate model including all risk factors, the only variable that remain significant was hypertension OR (IC95\%) of $2.5(1.1-5.7)(p=0.038)$.

\section{Discussion}

Main pathogens responsible for community-acquired uncomplicated UTIs were E. coli and ESBL-E. coli; however, the number of cases from the latter was lower compared to our own previously published findings (25\% versus 68\%) [11]. This discrepancy is highly due to differences in study design, since our previous published work was only based on laboratory reports database, and typically patients are only sent to the laboratory if they have recurrent infections or are not responding to treatment.

In the present work, we demonstrated an alarming resistance to commonly prescribed antibiotic drugs for uncomplicated UTIs (ampicillin, ceftriaxone, TMP-SMX, and ciprofloxacin).

When comparing our results to previous reports in other countries [22], we identified an even higher resistance to beta-lactams for ESBL-E. coli in our community. The presence of MDR genes including AmpC- $\beta$-lactamase, ESBLs, and carbapenemases is complicating the selection of empirical antimicrobial agents and is associated with treatment failure [23]. Previously published guidelines for UTIs recommend the initiation of treatment after a complete clinical evaluation and performance of urine analysis and cultures, for a judicious use of antibiotics, following local information on resistance patterns. There is also a general recommendation to avoid the use of antibiotics in asymptomatic bacteriuria; all of these measures will aid in a decreased exposition to antibiotics and avoid the rise in antimicrobial resistance rates $[8,24]$. In our study, the most common clinical finding was pollakiuria (64\%), and only $13 \%$ reported the presence of fever, which is consistent with a previous study, where the most frequent clinical finding was bladder tenderness in $93 \%$, followed by pollakiuria in $92 \%$, and the least common was fever in $7 \%$ of cases [7].

Some previously attributable risk factors for ESBLproducers infections are recurrence, older age, diabetes mellitus, female sex, urological procedures, prior use of antibiotics, anatomic anomalies, foreign material in the urinary tract, immunosuppression, and asymptomatic bacteriuria [23]. In our study, we confirmed diabetes in association with ESBL-E. coli, and even hypertension was associated with a higher strength. This association becomes remarkable, since we found two previous studies that reported E. coli bacteriuria with an increased risk for future hypertension development. Although the pathogenesis is not fully understood, there was a clear evidence of E. coli, a symptomatic bacteriuria implicated in the development of hypertension $[25,26]$. Hypertension role should be further investigated in association with ESBL-E. coli colonization. Limitations of the present study include recall bias, since we did not have access to complete medical records; also we were not able to register response to treatment in previous UTIs.

4.1. Considerations for UTIs Treatment. We found a low proportion of resistance to fosfomycin even in the ESBL producers, probably due to its limited use in the American continent [12]. This bactericidal agent is a phosphoenolpyruvate analogue that binds covalently to the amino acid residue Cys155 of MurA and prevents the peptidoglycan biosynthesis, important for bacterial cell wall formation [27]. Fosfomycin is currently recommended as first-line treatment for uncomplicated UTIs due to the capability to interfere with the formation of biofilms; it can be used in combination with quinolones or aminoglycosides to increase their therapeutic effects [8]. Furthermore, fosfomycin exerts immunomodulatory effects on monocytes and $\mathrm{T}$ and B lymphocytes via production of inflammatory cytokines; nevertheless, results from different studies have been contradictory [28]. However, it was established that neutrophils incubated with fosfomycin were capable of increasing their bactericidal ability when challenged with ESBL-producing $E$. coli [29], although resistance has also been reported lately by genetic mutations in the chromosomally encoded transport systems in Pseudomonas aeruginosa [30], which explains our findings of fosfomycin resistance in cases due to Pseudomonas, Klebsiella pneumoniae, and Proteus mirabilis; although we had a limited number of cases, one previous study found intermediate resistance to fosfomycin in 13 isolates of enterococci $(n=6), P$. aeruginosa $(n=5)$, and Klebsiella spp. $(n=2)$, and high resistance to fosfomycin in 5 isolates of M. morganii $(n=2), P$. aeruginosa $(n=1)$, Enterobacter aerogenes $(n=1)$, and Proteus mirabilis [31]. 
On the other hand, nitrofurantoin remains a reliable agent for the empirical treatment of uncomplicated UTIs since most uropathogens were shown to be sensible. However, its use has been discouraged specially for the elderly due to its potential for pulmonary toxicity as reported by the American Geriatrics Society Updated Beers Criteria [32]. Additionally, nitrofurantoin does not achieve adequate serum or tissue levels and therefore should not be used in severe disease and is also contraindicated in pregnancy and patients with renal failure [8]. TMP-SMX and quinolones should be discarded as first options for the treatment of community-acquired UTIs due to E. coli, since the overall resistance rates are consistently high (above 20\%) as recommended by the International Clinical Practice guidelines [12].

Furthermore, whenever there is suspicion of ESBL-E. coli, beta-lactams should be avoided, except for carbapenems and more recently developed beta-lactams such as ceftolozane-tazobactam and ceftazidime-avibactam [33]. In the case of Enterococcus faecalis, quinolones are a good therapeutic option in our media. Finally, carbapenems have the greatest level of activity against most uropathogens and have been recommended as first-line empiric therapy; nevertheless, we consider that their use should be restricted to serious infections, since its frequent use might be associated with colonization with carbapenem-resistant bacteria [34].

\section{Conclusion}

The most common pathogens being causative agents of community-acquired UTIs in our study were E. coli and ESBL-E. coli. For these, resistance rates to commonly prescribed antibiotic drugs were high, with the exception of fosfomycin, nitrofurantoin, and carbapenems. For other pathogens such as Klebsiella and Proteus, resistance rates to TMP-SMX and quinolones were low. Hypertension and diabetes were significantly associated with ESBL-E. coli in comparison to ESBL-non-producers. As a general recommendation, the identification of additional risk factors, such as the presence of diabetes or hypertension, could yield in a better selection of treatment, along with pathogen identification. Our results demonstrate that an active surveillance of resistance patterns in UTIs in the community is required since the proportion of ESBL producers represents a warning. Finally, the current use of fosfomycin in the American continent might be hampered due to lack of local in vitro susceptibility data and clinical experience; we expect that with the data provided in here, more clinical trials can be conducted to support and further explore the information presented.

\section{Data Availability}

The data used to support the findings of this study are included within the article and within the supplementary information file(s).

\section{Ethical Approval}

The study was approved by the Health Sciences Institutional Review Board.

\section{Consent}

All patients provided their consent to be involved in the study, and their personal information was kept confidential always during the study.

\section{Conflicts of Interest}

The authors declare no conflicts of interest.

\section{Authors' Contributions}

(1) MGZC, IZT, and FAST conceptualized the study; (2) MGZC, JARC, and RG were responsible for methodology; (3) JARC, FAST, and RG validated the study, (4) MSC, SFNP, and MGZC were responsible for formal analysis, investigation, and data curation, and (5) MSC, RG, IZT, SFNP, JARC, FAST, and MGZC were responsible for original draft preparation, review, and editing.

\section{Acknowledgments}

The study was funded (urinalysis, urine cultures, laboratory supplies, and salary for general practitioners) by a research grant from Laboratorios Senosiain SA de CV which is a company that commercializes ciprofloxacin, levofloxacin, and fosfomycin in Mexico. MGZC and IZT received funding from Laboratorios Senosiain, including transportation and housing to attend international meetings. The pharmaceutical company had no role in the study design, data collection, analysis, and preparation of the manuscript.

\section{Supplementary Materials}

Supplementary Table 1: main isolated pathogens $(n \geq 4)$ in urine samples from patients with community-acquired UTIs and their antimicrobial patterns. (Supplementary Materials)

\section{References}

[1] B. Foxman, "The epidemiology of urinary tract infection," Nature Reviews Urology, vol. 7, no. 12, pp. 653-660, 2010.

[2] S. A. Ferry, S. E. Holm, H. Stenlund, R. Lundholm, and T. J. Monsen, "Clinical and bacteriological outcome of different doses and duration of pivmecillinam compared with placebo therapy of uncomplicated lower urinary tract infection in women: the LUTIW project," Scandinavian Journal of Primary Health Care, vol. 25, no. 1, pp. 49-57, 2007.

[3] L. E. Nicolle, "Asymptomatic bacteriuria-important or not?" New England Journal of Medicine, vol. 343, no. 14, pp. 1037-1039, 2000.

[4] S. E. Geerlings, "Clinical presentations and epidemiology of urinary tract infections," Microbiology Spectrum, vol. 4, no. 5, 2016.

[5] F. Yeganeh-Sefidan, R. Ghotaslou, M. Akhi et al., "Fosfomycin, interesting alternative drug for treatment of urinary tract infections created by multiple drug resistant and extended spectrum beta-lactamase producing strains," Iranian Journal of Microbiology, vol. 8, no. 2, pp. 125-131, 2016.

[6] E. J. Dielubanza and A. J. Schaeffer, "Urinary tract infections in women," Medical Clinics of North America, vol. 95, no. 1, pp. 27-41, 2011. 
[7] A. E. Barber, J. P. Norton, A. M. Spivak, and M. A. Mulvey, "Urinary tract infections: current and emerging management strategies," Clinical Infectious Diseases, vol. 57, no. 5, pp. 719-724, 2013.

[8] K. Gupta and N. Bhadelia, "Management of urinary tract infections from multidrug-resistant organisms," Infectious Disease Clinics of North America, vol. 28, no. 1, pp. 49-59, 2014.

[9] L. Rossignol, S. Vaux, S. Maugat et al., "Incidence of urinary tract infections and antibiotic resistance in the outpatient setting: a cross-sectional study," Infection, vol. 45, no. 1, pp. 33-40, 2017.

[10] Y. Doi, Y. S. Park, J. I. Rivera et al., "Community-associated extended-spectrum beta-lactamase-producing Escherichia coli infection in the United States," Clinical Infectious Diseases, vol. 56, no. 5, pp. 641-648, 2013.

[11] M. G. Zavala-Cerna, N. Macriz-Romero, J. F. SantoscoyGutierrez et al., "High microbiological spectrum resistance rates in urine isolates from Jalisco, Mexico. A retrospective study and literature review," International Archives of Medicine, vol. 8, no. 148, 2015.

[12] K. Gupta, T. M. Hooton, K. G. Naber et al., "International clinical practice guidelines for the treatment of acute uncomplicated cystitis and pyelonephritis in women: a 2010 update by the infectious diseases society of America and the European society for microbiology and infectious diseases," Clinical Infectious Diseases, vol. 52, no. 5, pp. e103-e120, 2011.

[13] A. Colpan, B. Johnston, S. Porter et al., "Escherichia coli sequence type 131 (ST131) subclone $\mathrm{H} 30$ as an emergent multidrug-resistant pathogen among US veterans," Clinical Infectious Diseases, vol. 57, no. 9, pp. 1256-1265, 2013.

[14] E. Voulgari, A. Poulou, V. Koumaki, and A. Tsakris, "Carbapenemase-producing enterobacteriaceae: now that the storm is finally here, how will timely detection help us fight back?" Future Microbiology, vol. 8, no. 1, pp. 27-39, 2013.

[15] R. Roy, M. Tiwari, G. Donelli, and V. Tiwari, "Strategies for combating bacterial biofilms: a focus on anti-biofilm agents and their mechanisms of action," Virulence, vol. 9, no. 1, pp. 522-554, 2018.

[16] I. Roca, M. Akova, F. Baquero et al., "The global threat of antimicrobial resistance: science for intervention," New Microbes and New Infections, vol. 6, pp. 22-29, 2015.

[17] J. O’Neill, “Tackling drug-resistant infections globally: final report and recommendations," The Review on Antimicrobial Resistance, pp. 4-5, 2016, https://amr-review.org/sites/ default/files/AMR\%20Review\%20Paper\%20-\%20Tackling\% 20 a $\% 20$ crisis $\% 20$ for $\% 20$ the $\% 20$ health $\% 20$ and $\% 20$ wealth $\%$ 20of\%20nations_1.pdf.

[18] J. A. Ayukekbong, M. Ntemgwa, and A. N. Atabe, "The threat of antimicrobial resistance in developing countries: causes and control strategies," Antimicrobial Resistance \& Infection Control, vol. 6, no. 1, p. 47, 2017.

[19] T. L. Holland, C. W. Woods, and M. Joyce, "Antibacterial susceptibility testing in the clinical laboratory," Infectious Disease Clinics of North America, vol. 23, no. 4, pp. 757-790, 2009.

[20] Clinical and Laboratory Standards Institute, Inhibitory Concentrations of Aerobic Bacteria by Broth Macrodilution, and Agar Dilution, Methods for Dilution Antimicrobial Susceptibility Tests for Bacteria that Grow Aerobically; Approved Standard, in Clinical and Laboratory Standards Institute, Wayne, PA, USA, 9th edition, 2015.

[21] I. Wiegand, K. Hilpert, and R. E. W. Hancock, "Agar and broth dilution methods to determine the minimal inhibitory concentration (MIC) of antimicrobial substances," Nature Protocols, vol. 3, no. 2, pp. 163-175, 2008.

[22] R. Bonnet, "Growing group of extended-spectrum $\beta$-lactamases: the CTX-M enzymes," Antimicrobial Agents and Chemotherapy, vol. 48, no. 1, pp. 1-14, 2004.

[23] S. Meier, R. Weber, R. Zbinden, C. Ruef, and B. Hasse, "Extended-spectrum $\beta$-lactamase-producing Gram-negative pathogens in community-acquired urinary tract infections: an increasing challenge for antimicrobial therapy," Infection, vol. 39, no. 4, pp. 333-340, 2011.

[24] A. Silva Filho, S. Contreras, and J. Haddad, "Recurrent urinary tract infection in the gynecologic practice: time for reviewing concepts and management," Revista Brasileira de Ginecologia e Obstetrícia/RBGO Gynecology and Obstetrics, vol. 39, no. 1, pp. 1-3, 2017.

[25] R. Meiland, S. E. Geerlings, R. P. Stolk et al., "Escherichia coli bacteriuria in female adults is associated with the development of hypertension," International Journal of Infectious Diseases, vol. 14, no. 4, pp. e304-e307, 2010.

[26] R. Meiland, R. P. Stolk, S. E. Geerlings et al., "Association between Escherichia coli bacteriuria and renal function in women," Archives of Internal Medicine, vol. 167, no. 3, pp. 253-257, 2007.

[27] F. Docobo-Pérez, G. L. Drusano, A. Johnson et al., "Pharmacodynamics of fosfomycin: insights into clinical use for antimicrobial resistance," Antimicrobial Agents and Chemotherapy, vol. 59, no. 9, pp. 5602-5610, 2015.

[28] A. S. Michalopoulos, I. G. Livaditis, and V. Gougoutas, "The revival of fosfomycin," International Journal of Infectious Diseases, vol. 15, no. 11, pp. e732-e739, 2011.

[29] R. Krause, S. Patruta, F. Daxböck, P. Fladerer, and C. Wenisch, "The effect of fosfomycin on neutrophil function," Journal of Antimicrobial Chemotherapy, vol. 47, no. 2, pp. 141-146, 2001.

[30] Z. Beharry and T. Palzkill, "Functional analysis of active site residues of the fosfomycin resistance enzyme FosA from Pseudomonas aeruginosa," Journal of Biological Chemistry, vol. 280, no. 18, pp. 17786-17791, 2005.

[31] E. B. Hirsch, B. R. Raux, P. C. Zucchi et al., "Activity of fosfomycin and comparison of several susceptibility testing methods against contemporary urine isolates," International Journal of Antimicrobial Agents, vol. 46, no. 6, pp. 642-647, 2015.

[32] American Geriatrics Society Beers Criteria Update Expert Panel, "American geriatrics society updated beers criteria for potentially inappropriate medication use in older adults," Journal of the American Geriatrics Society, vol. 60, no. 4, pp. 616-631, 2012.

[33] D. van Duin and R. A. Bonomo, "Ceftazidime/avibactam and ceftolozane/tazobactam: second-generation $\beta$-lactam/ $\beta$-lactamase inhibitor combinations," Clinical Infectious Diseases, vol. 63, no. 2, pp. 234-241, 2016.

[34] M. S. Bader, M. Loeb, and A. A. Brooks, "An update on the management of urinary tract infections in the era of antimicrobial resistance," Postgraduate Medicine, vol. 129, no. 2, pp. 242-258, 2017. 